\title{
SPECTROSCOPIC OBSERVATIONS OF THE
}

\section{Be STARS $\boldsymbol{\eta}$ Cen, $\gamma$ Cas AND $\phi$ Per}

\author{
W. M. BURTON and R. G. EVANS \\ Appleton Laboratory (Astrophysics Research Division) \\ Culham Laboratory, Abingdon, Oxfordshire, England
}

\section{Introduction}

The main part of this paper is concerned with observations obtained using the Princeton ultraviolet telescope-spectrometer on the Copernicus satellite through the Princeton guest investigator programme. These observations provided high resolution ultraviolet spectra of the bright southern shell star $\eta$ Cen, which are now being analysed in detail. A short description of the new $\eta$ Cen spectra and a progress report on the data analysis are presented below. In the second part of the paper we describe visible-region spectra of the northern Be stars $\gamma$ Cas and $\phi$ Per obtained with a cross-dispersed echelle spectrograph on the 36-inch Yapp telescope at the Royal Greenwich Observatory.

\section{Copernicus Ultraviolet Spectra of $\eta$ Cen (HD 127972)}

The Princeton telescope-spectrometer on the Copernicus satellite (Rogerson et al., 1973) consists of a Cassegrain telescope of $0.8 \mathrm{~m}$ aperture with a concave grating spectrometer which has four scanning photomultiplier detectors. Two of these detectors, designated $U 1$ and $U 2$, scan the second order spectrum in the wavelength range $\lambda 750 \AA-1500 \AA$ giving spectral resolutions of $0.05 \AA$ and $0.2 \AA$ respectively. Two other detectors, designated V1 and V2, simultaneously scan the first order spectrum in the wavelength range $\lambda 1500 \AA-3000 \AA$ with spectral resolutions of $0.1 \AA$ and $0.4 \AA$. At each discrete point in the wavelength scan, the detectors pause for 14 seconds and record an integrated count for this time interval.

The observations of $\eta$ Cen were obtained on 1-2 March 1975 and included two scans in the wavelength range $\lambda 1000 \AA$ to $1435 \AA$ made with the U2 detector $(d \lambda \simeq 0.2 \AA)$. Simultaneously, the U1 detector scanned selected spectral regions at high resolution $(d \lambda=0.05 \AA)$ to record individual spectral features, including resonance absorption lines of $\mathrm{N}_{\mathrm{I}}, \mathrm{N}_{\mathrm{II}}, \mathrm{S}_{\mathrm{II}}, \mathrm{Fe}_{\mathrm{II}}$ and $\mathrm{Fe}$ III. Additionally, data were also obtained with the V2 detector in the wavelength range $\lambda 2000 \AA$ to $2870 \AA$ but these observations are not discussed in the present paper.

The new ultraviolet observations of $\eta$ Cen are generally similar to previously observed spectra of normal stars of comparable spectral type, no emission lines being detected in the ultraviolet region. In the wavelength range $\lambda 1000 \AA$ to $1430 \AA$ the spectrum of $\eta$ Cen shows considerable line blanketing, the predominant ions being Si III, Cr III and Fe III. Many of the observed spectral features show doppler broadened profiles resulting from the high rotational velocity which is considerably 


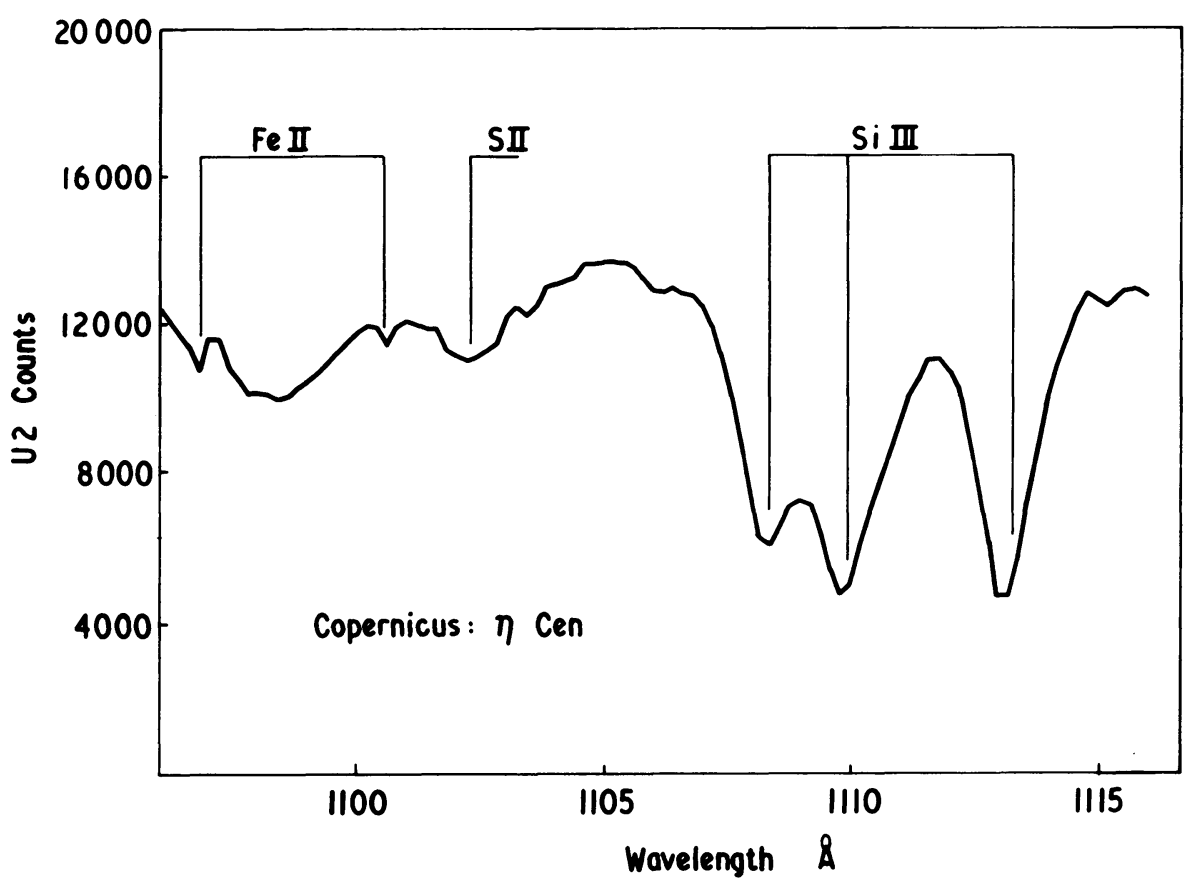

Fig. 1. Copernicus ultraviolet spectrum of $\eta$ Cen (U2 scan $\lambda 1096 \AA-1115 \AA$ ) showing absorption lines of Si III, S II and Fe II.

larger than the instrumental resolution $\left(\simeq 50 \mathrm{~km} \mathrm{~s}^{-1}\right)$. Figure 1 shows part of the $\mathrm{U} 2$ scan between $\lambda 1096 \AA$ and $1115 \AA$ which includes absorption lines of Si III, S II and $\mathrm{Fe}$ II. The well developed triplet of Si III is almost certainly formed near the stellar surface and is broadened both by rotation and by radiative damping. The weak $S_{\text {II }}$ line at $\lambda 1102 \AA$ shows the dish-shaped profile characteristic of rotational broadening, giving a projected rotational velocity $v \sin i \simeq 190 \mathrm{~km} \mathrm{~s}^{-1}$. Table I gives a summary of similar measurements on other weak and unblended rotationbroadened lines in the ultraviolet which provide velocity data for a wide range of ions.

TABLE I

Measured rotational velocities in the $\eta$ Cen spectrum

\begin{tabular}{lll}
\hline Ion & Wavelength $(\AA)$ & $v \sin i\left(\mathrm{~km} \mathrm{~s}^{-1}\right)$ \\
\hline S II $_{\text {II }}$ & 1102.3 & 190 \\
S IIV $_{\text {IV }}$ & 1077.1 & 220 \\
P III $_{\text {Fe III }}$ & 1062.7 & 200 \\
Fe III & 1068.6 & 160 \\
Fe III & 1124.9 & 140 \\
Cr III & 1126.7 & 210 \\
Cr III & 1132.9 & 160 \\
\hline
\end{tabular}


Observations of $\eta$ Cen in the visible region give a value of $v \sin i \simeq 300 \mathrm{~km} \mathrm{~s}^{-1}$ for absorption lines formed near the stellar photospheric surface (Uesugi and Fukuda, 1970). This velocity is greater than the measured values for the ultraviolet lines listed in Table I, giving some evidence of a progression from this high value near to the stellar surface through intermediate values ( $\mathrm{Cr}$ III, $\mathrm{S}_{\text {III, }} \mathrm{S}_{\text {III }}$ ) to the much smaller value $\left(\sim 140 \mathrm{~km} \mathrm{~s}^{-1}\right)$ observed for those absorption lines (e.g. P III) which are probably formed further out in the differentially rotating extended atmosphere. If it is assumed that angular momentum is conserved in this differentially rotating atmosphere, then these observations suggest that the envelope has an equatorial radius $R_{e} \simeq 2 R_{*}$.

Visible spectrum observations of $\eta$ Cen have been discussed by Jaschek et al. (1964) who describe it as a shell star of spectral type B1.5 Vne. The H $\alpha$ line was observed as a single broad emission feature in 1960 but one year later the line became double. This variability was also present in $\mathrm{H} \beta$ and $\mathrm{H} \gamma$ which have changed from double emission lines to wide emission features with sharp central absorption cores during the period 1959-1962.

In contrast to this earlier classification of $\boldsymbol{\eta}$ Cen as a shell star, the new ultraviolet observations provide little evidence for the presence of a shell spectrum. The lines of Fe II at $1097 \AA$ and $1101 \AA$ which are shown in Figure 1 are very weak and narrow, having widths equal to the limiting instrumental spectral resolution $\left(\sim 50 \mathrm{~km} \mathrm{~s}^{-1}\right)$. These lines would be expected to be much stronger and broader if formed in a circumstellar shell and they are most probably of interstellar origin, being in good agreement with the observations of interstellar absorption lines in normal B-type stars at similar distances (Rogerson et al., 1973). Further evidence for the extreme weakness of possible shell features is obtained from the profiles of the $\mathrm{N}_{\text {I }}$ triplet at $\lambda 1134 \AA$ and the $N_{\text {II line at }} \lambda 1084 \AA$. Figure 2 shows the $N_{I}$ lines which are extremely narrow $\left(d \lambda \simeq 0.1 \AA\right.$ or $\left.25 \mathrm{~km} \mathrm{~s}^{-1}\right)$, their width and intensity being consistent with purely interstellar absorption. The $\lambda 1084 \AA \mathrm{N}_{\text {II }}$ line appears as a very narrow feature $\left(d \lambda \simeq 0.1 \AA\right.$ ) within the much broader stellar $\mathrm{N}_{\text {II }}$ multiplet and is again consistent with purely interstellar absorption. However, some evidence for the existence of a low density shell is seen in Figure 3 which shows a U2 scan from $\lambda 1188 \AA$ to $1208 \AA$. The dominant absorption lines are due to a multiplet of $S$ in but the more interesting features are the two pairs of $\mathrm{Si}$ II lines, one originating from the ground state $\left(0 \mathrm{~cm}^{-1}\right)$ and the other from a very low-lying excited fine structure state $\left(287 \mathrm{~cm}^{-1}\right)$.

The narrow absorption line at $\lambda 1193 \AA$ corresponds to the transition from the ground state and is probably interstellar but the $\lambda 1198 \AA$ line which originates from the excited $287 \mathrm{~cm}^{-1}$ level is much broader. The $\lambda 1198 \AA$ line is not normally seen in the interstellar gas since it requires a density $n_{\mathrm{H}} \simeq 10^{3}$ atom $\mathrm{cm}^{-3}$ to populate this fine structure level (Bahcall and Wolf, 1968). It may be that this absorption occurs in a shell or circumstellar envelope with a gas density $\geq 10^{3}$ atom $\mathrm{cm}^{-3}$, but the possibility remains that it could be a weak stellar feature, its profile being generally

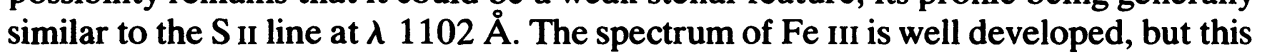
does not necessarily indicate the presence of a shell, since the profiles of the ultraviolet multiplets of Fe III in the present spectrum are wholly consistent with their being of stellar origin. 


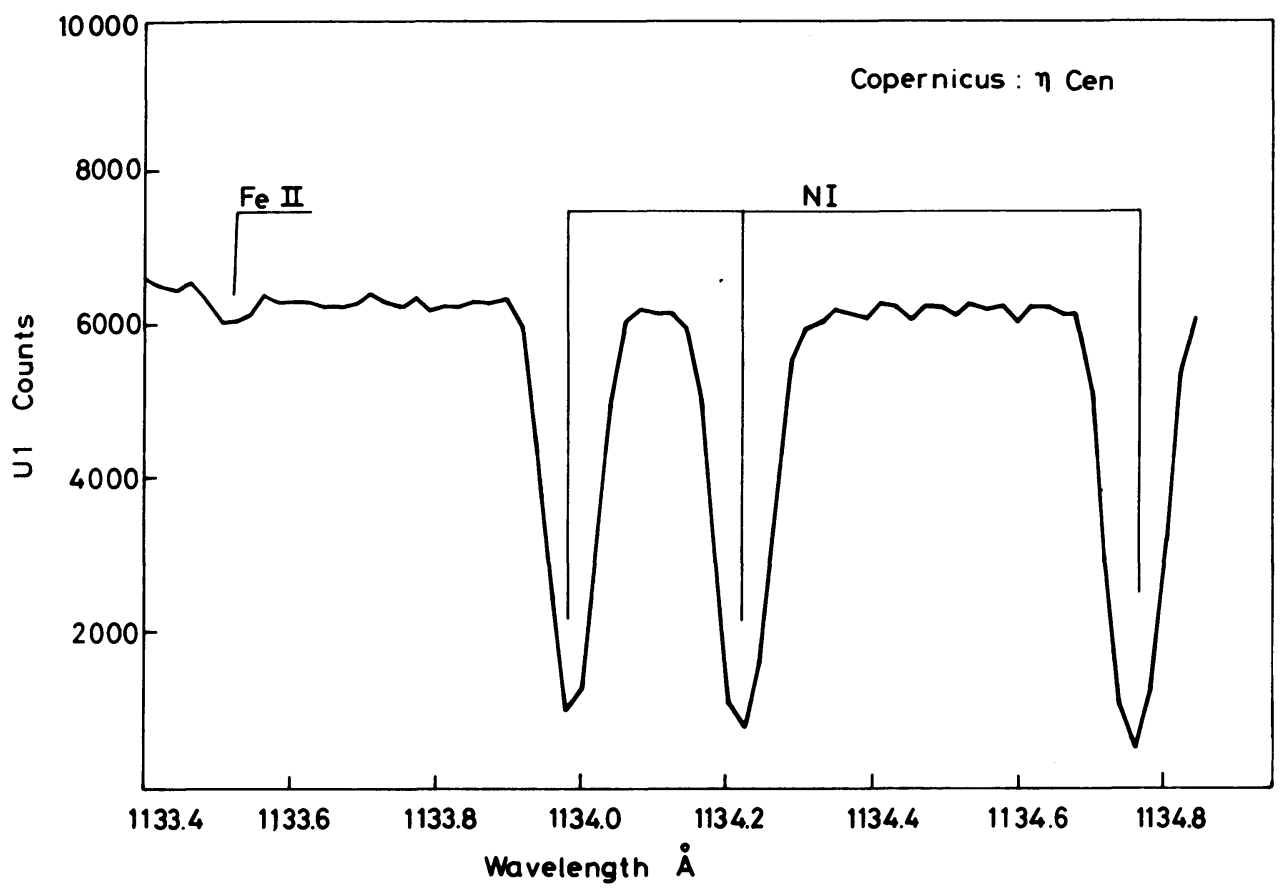

Fig. 2. Copernicus ultraviolet spectrum of $\eta$ Cen (U1 scan $\lambda 1133.4 \AA-1134.8 \AA$ ) showing interstellar lines of $\mathrm{N} \mathrm{I}$ and $\mathrm{Fe}$ II.

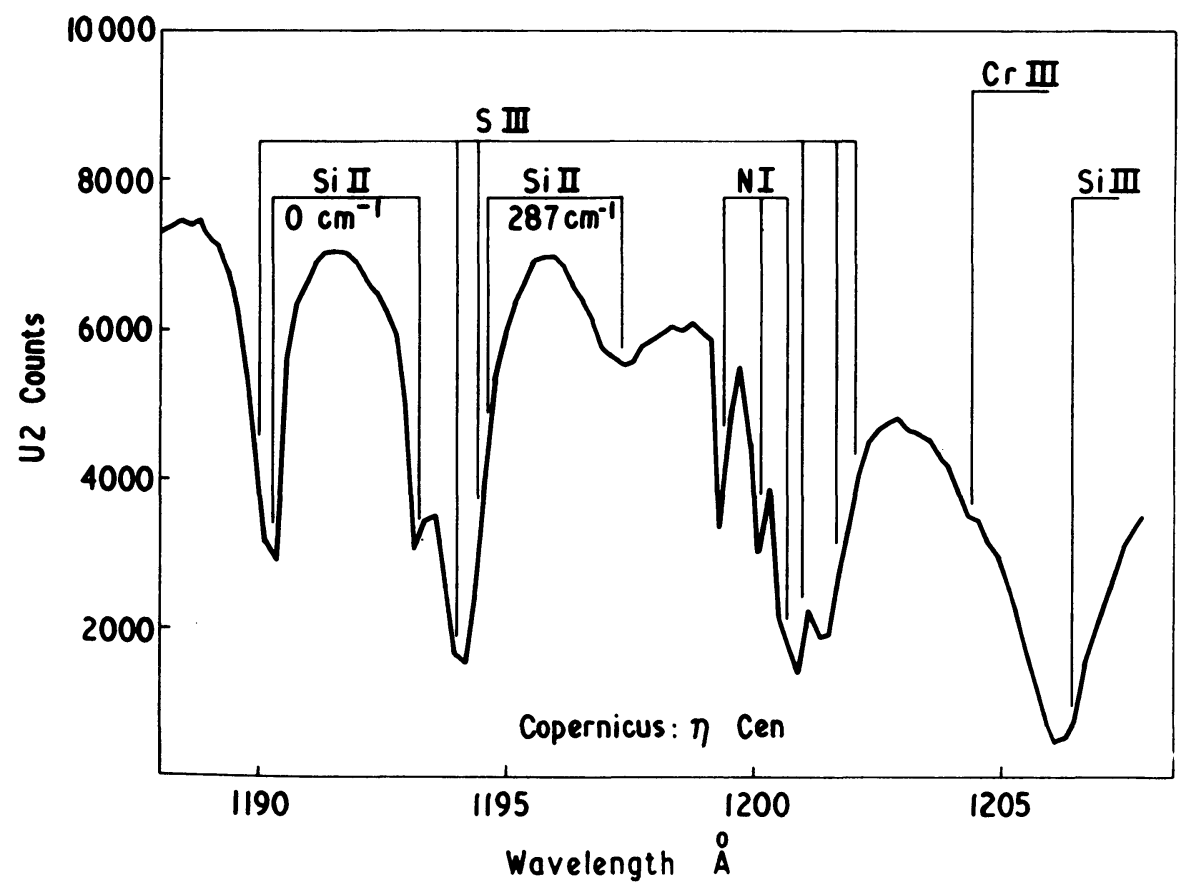

Fig. 3. Copernicus ultraviolet spectrum of $\eta$ Cen (U2 scan $\lambda 1188 \AA-1208 \AA$ ) showing absorption lines from the ground level and excited levels in $\mathrm{Si}$ II. 


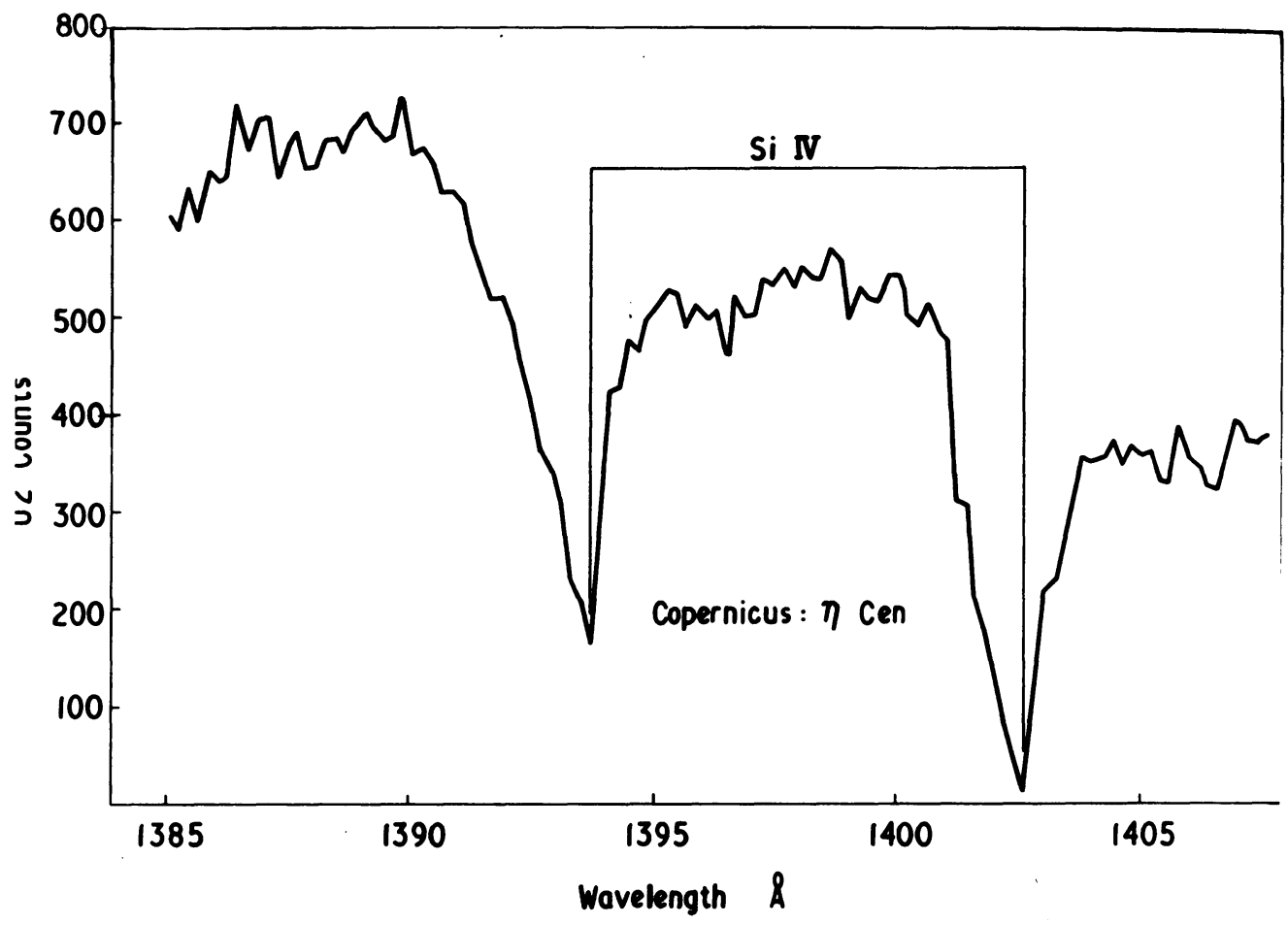

Fig. 4. Copernicus ultraviolet spectrum of $\eta$ Cen (U2 scan $\lambda 1385 \AA-1408 \AA$ ) showing the resonance doublet of $\mathrm{Si}$ IV with asymmetric absorption line profiles indicating high expansion velocities.

Another feature of interest in the $\eta$ Cen ultraviolet spectrum is illustrated in Figure 4 which shows the U2 scan in the region of the Si IV resonance lines near $\lambda 1400 \AA$. The continuum level in this tracing has been distorted by an incomplete correction for stray light in the spectrometer which has resulted in an incorrect stepped continuum level. The absorption lines are in fact saturated and should reach the zero intensity level. Both components of the Si IV multiplet show asymmetric profiles with an enhanced short wavelength wing, giving a limiting velocity value of about $-250 \mathrm{~km} \mathrm{~s}^{-1}$. Blue-shifted absorption lines are normally interpreted in terms of mass outflow from the star, which would result in mass-loss if the flow velocity exceeds the local escape velocity. The predicted escape velocity at the surface of a normal B2 V star is estimated to be $\sim 700 \mathrm{~km} \mathrm{~s}^{-1}$ but for $\eta$ Cen, which has a rapidly rotating extended atmosphere, the escape velocity from this atmosphere will be significantly lower. If we assume that the observed Si IV expansion velocity in $\boldsymbol{\eta}$ Cen is in fact resulting in mass loss and that the $\mathrm{Si}$ abundance is normal, then the mass loss rate is calculated to be $\sim 2 \times 10^{-11} M_{\odot} \mathrm{y}^{-1}$. This value is based on the assumption that the mass outflow is approximately spherical and it would be reduced by about an order of magnitude if equatorial flow predominated. This estimated mass loss rate from $\eta$ Cen is less by a factor of $\sim 10^{-5}$ than from Wolf Rayet and Of stars, but if this value is typical, then the larger number of Be stars may make them an important factor in determining the chemical balance of the interstellar medium.

Although the analysis of the new ultraviolet observations is by no means complete at the present time, it is nevertheless possible to draw a few preliminary conclusions. 
There is good evidence that the atmosphere of $\eta$ Cen is extended and rapidly rotating. The Si IV lines provide very strong evidence for the existence of an expanding atmosphere and possibly for mass loss. The existence of a circumstellar shell is less certain, but there does seem to be some indication of a significant density $\left(\geq 10^{3} \mathrm{~cm}^{-3}\right)$ at a high level in the extended stellar atmosphere. However, the strong absorption lines of ions such as Fe II, which are normally associated with shell spectra, do not seem to occur in the ultraviolet spectrum of $\eta$ Cen suggesting that there is no strongly developed shell at the present time.

\section{Visible Region Spectra of $\gamma$ Cas (HD 5394) and $\phi$ Per (HD 10516)}

The two bright Be stars $\gamma$ Cas and $\phi$ Per were designated as stars of particular interest for the present symposium and it was suggested that new observations would be valuable. For this reason spectra of both stars were recorded on 7-8 June 1975, using the 36-in. Yapp telescope at the Royal Greenwich Observatory. The primary aim during this observing run was to evaluate the performance of a recently developed image tube echelle spectrograph which combines good spectral resolution with wide wavelength coverage. The spectrograph was originally designed for use in rocket vehicles to observe ultraviolet stellar spectra (Burton et al., 1971) but was subsequently converted for use with ground-based telescopes. In its new configuration (CRESTA) this spectrograph provides a dispersion of $\sim 5 \AA \mathrm{mm}^{-1}$ (spectral resolution $d \lambda \widetilde{1.0} \AA$ ) over a wavelength range extending from the image tube window limit $(\approx \lambda 3800 \AA)$ up to the sensitivity limit of the S25 photocathode $(\sim \lambda 8000 \AA)$. The optical system consists of a concave collimator mirror, echelle grating, plane cross-dispersing grating for order separation and a concave camera mirror which focuses the spectrum on to the input window of a Westinghouse WL30677 image intensifier. Photographic film (Kodak 103aD or 103aF) is contacted against the output fibre optic of this image tube to record the intensified spectra.

An example of the two-dimensional spectrum format produced by this crossdispersed echelle grating system is shown in Figure 5. This spectrum of $\phi$ Per $\left(\mathrm{B} 0 \mathrm{e} m_{v}=4.2\right)$ was obtained in a 10 minute exposure using Kodak 103aF film. One advantage of the large area echelle format for the study of $\mathrm{Be}$ stars is at once apparent. The extended wavelength coverage enables the entire Balmer series to be recorded in a single exposure while the echelle dispersion provides sufficient spectral resolution to record the detailed profiles of the various emission lines, thus permitting simultaneous study of spectrum changes in the different members of the Balmer series. Observations of this type were made for both $\gamma$ Cas and $\phi$ Per on 7-8 June 1975 and Figure 6 shows densitometer traces of the profiles of $\mathrm{H} \alpha$ and $\mathrm{H} \beta$ emission lines in these two stars. Further data reduction will be necessary to correct for the instrument sensitivity functions but nevertheless the observations show the general shape of the emission line profiles.

The general form of the observed profiles is similar to those obtained by Gray and Marlborough (1974) using a photoelectric line-profile scanner. The $\mathrm{H} \alpha$ emission line in $\phi$ Per does not show appreciable splitting but the $\mathrm{H} \beta$ line has a pronounced 


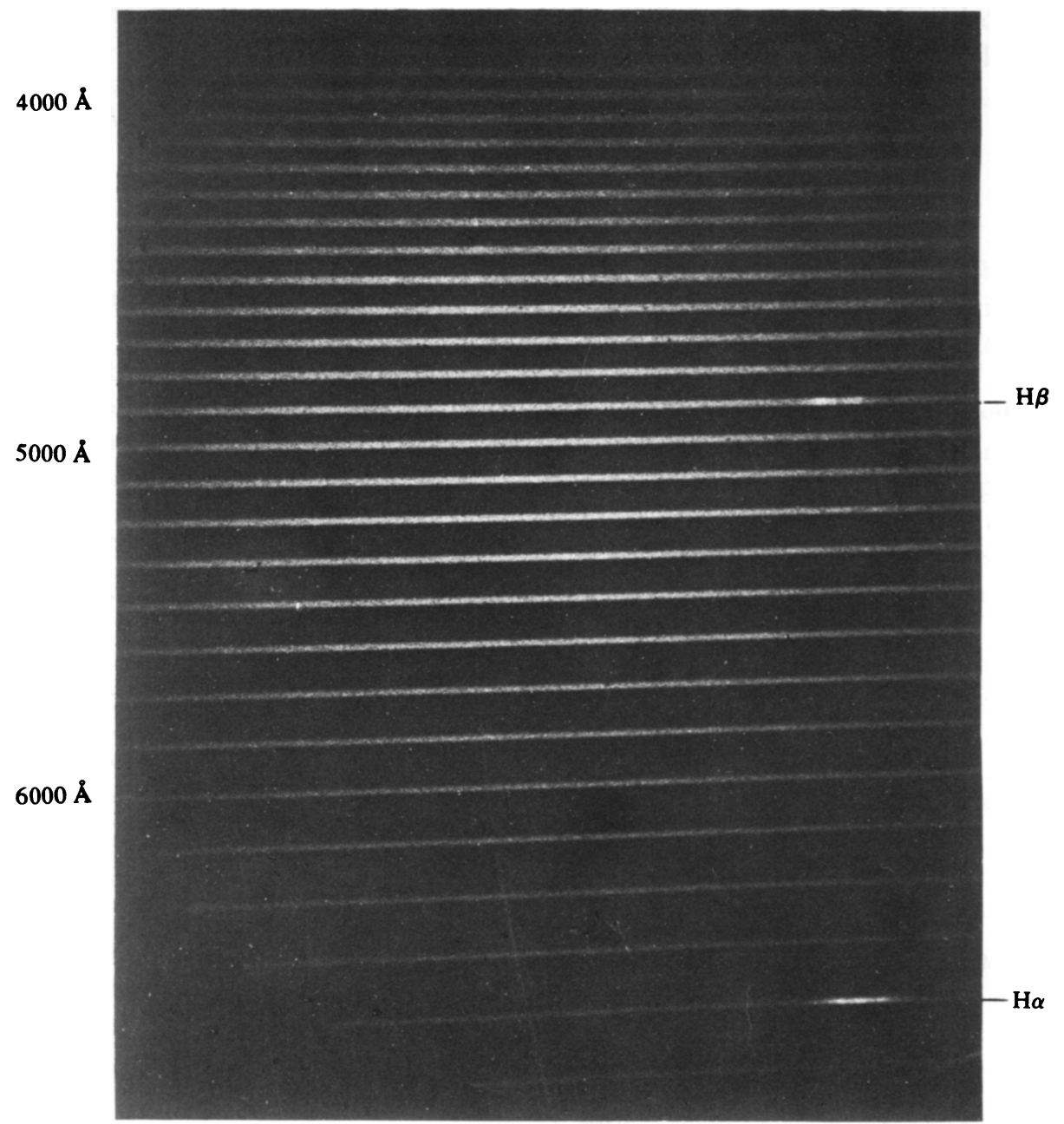

$7000 \AA$

Fig. 5. Echelle spectrum of $\phi \operatorname{Per}\left(B 0 e m_{v}=4.2\right)$ recorded with the CRESTA spectrograph on the RGO 36-in. telescope (cassegrain focus). 7-8 June 1975. Exposure $10 \mathrm{~min}$ on 103aF film.

double peak with $V<R$. In $\gamma$ Cas the $\mathrm{H} \alpha$ line is double and has the same general shape as the double-peaked emission in $\mathrm{H} \beta$ but in this case $V>R$. If the observed $V / R$ ratio is interpreted as the result of a wavelength shift of a central absorption component, then we might conclude that the absorbing atmospheric material in $\phi$ Per is expanding at the present time while that in $\gamma$ Cas, being red-shifted, is falling back towards the underlying star.

The profiles illustrate an important point in the common terminology associated with Be star spectra. It was suggested in an earlier paper at this symposium (Bidelman, 1975) that a shell star can be recognised by the presence of Balmer emission lines with central absorption features. In the case of $\gamma$ Cas and $\phi$ Per the 

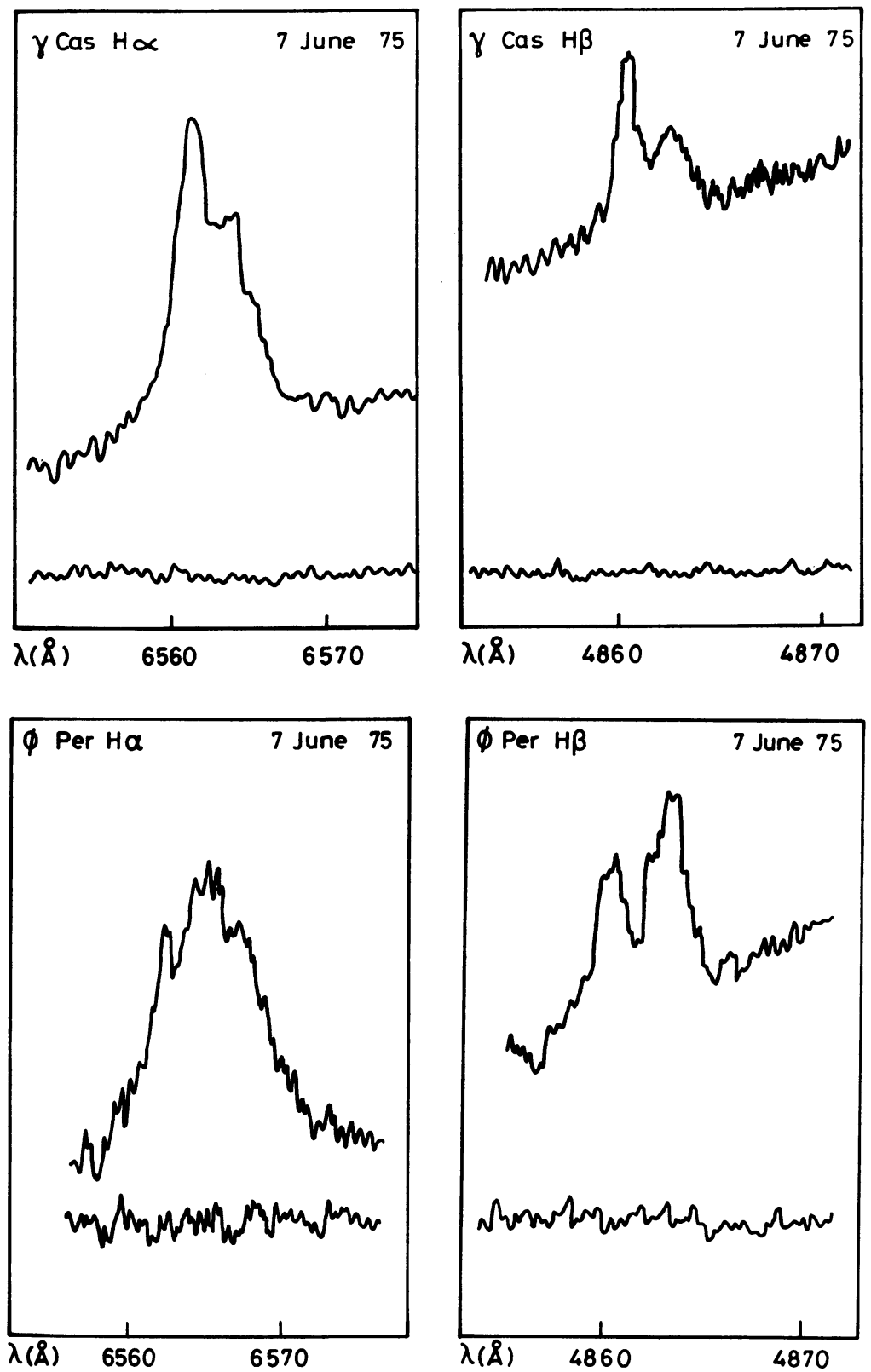

Fig. 6. Densitometer tracings of $\mathrm{H} \alpha$ and $\mathrm{H} \beta$ emission lines in $\gamma$ Cas and $\phi$ Per from echelle spectrograph exposures with the RGO 36-in. telescope on 7-8 June 1975.

Balmer lines indeed show double emission peaks but in neither case does the absorption minimum extend below the local stellar continuum level, so that in terms of the stellar continuum this central 'absorption' is still an emission feature. A possible criterion which could be adopted for consistency would be to designate as 
'shell stars' only those stars with distinct shell absorption lines which depress the stellar flux below the local continuum level. The Balmer line profiles obtained in the present observations would then be described as double emission peaks and would not necessarily indicate the presence of a shell.

\section{Acknowledgements}

The Copernicus observations described in the first part of this paper were obtained through the generosity of Professor L. Spitzer and his colleagues at Princeton University Observatory. In particular we thank Dr D. York and Dr T. Snow for their help in obtaining the observations.

The observations made with the 36-inch Yapp telescope were obtained with the permission of the Director of the Royal Greenwich Observatory. Important contributions made by Mr R. A. Hardcastle and other members of the Appleton Laboratory Astrophysics Research Division during the modification of the echelle spectrograph for the ground-based observations are gratefully acknowledged. This paper is published with the permission of the Director of the Appleton Laboratory.

\section{References}

Bahcall, J. N. and Wolf, R. A.: 1968, Astrophys. J. 152, 701.

Burton, W. M., Reay, N. K., Shenton, D. B., and Wilson, R.: 1971, in F. Läbuhn and R. Lüst (eds.), 'New Techniques in Space Astronomy', IAU Symp. 41, 304.

Gray, D. F. and Marlborough, J. M.: 1974, Astrophys. J. Suppl. 27, 121.

Jaschek, C., Jaschek, M., and Kucewicz, B.: 1964, Z. Astrophys. 59, 108.

Rogerson, J. B., York, D. G., Drake, J. F., Jenkins, E. B., Morton, D. C., and Spitzer, L.: 1973, Astrophys. J. Letters 181, L 110.

Uesugi, A. and Fukuda, I.: 1970, Contrib. Inst. Astrophys and Kwasan Obs., Univ. of Kyoto, No. 189. 